\title{
CDK4/6 inhibition is more active against the glioblastoma proneural subtype
}

\author{
Ming Li ${ }^{1,2}$, Aizhen Xiao ${ }^{1}$, Desiree Floyd ${ }^{1}$, Inan Olmez ${ }^{1}$, Jeongwu Lee ${ }^{3}$, Jakub \\ Godlewski ${ }^{4}$, Agnieszka Bronisz ${ }^{4}$, Krishna P.L. Bhat ${ }^{5}$, Erik P. Sulman ${ }^{6}$, Ichiro \\ Nakano' and Benjamin Purow ${ }^{1}$ \\ ${ }^{1}$ Neuro-Oncology Division, Department of Neurology, University of Virginia, Charlottesville, VA, USA \\ ${ }^{2}$ The Experiment Center, The Second Affiliated Hospital of Soochow University, Suzhou, Jiangsu, China \\ ${ }^{3}$ Department of Stem Cell Biology and Regenerative Medicine, Lerner Research Institute, Cleveland Clinic, Cleveland, OH, \\ USA \\ ${ }^{4}$ Department of Neurosurgery, Brigham and Women's Hospital, Boston, MA, USA \\ ${ }^{5}$ Departments of Pathology, MD Anderson Cancer Center, Houston, TX, USA \\ ${ }^{6}$ Radiation Oncology, MD Anderson Cancer Center, Houston, TX, USA \\ ${ }^{7}$ Department of Neurosurgery, University of Alabama, Birmingham, AL, USA \\ Correspondence to: Benjamin Purow, email: bwp5g@virginia.edu
}

Keywords: glioblastoma, palbociclib, proneural, mesenchymal, CDK4/6

Received: March 10, $2017 \quad$ Accepted: July 12, $2017 \quad$ Published: July 21, 2017

Copyright: Li et al. This is an open-access article distributed under the terms of the Creative Commons Attribution License 3.0 (CC BY 3.0), which permits unrestricted use, distribution, and reproduction in any medium, provided the original author and source are credited.

\section{ABSTRACT}

Glioblastoma (GBM) is the most common and lethal brain tumor. Gene expression profiling has classified GBM into distinct subtypes, including proneural, mesenchymal, and classical, and identifying therapeutic vulnerabilities of these subtypes is an extremely high priority. We leveraged The Cancer Genome Atlas (TCGA) data, in particular for microRNA expression, to seek druggable core pathways in GBM. The E2F1-regulated miR-17 92 cluster and its analogs are shown to be highly expressed in proneural GBM and in GSC lines, suggesting the E2F cell cycle pathway might be a key driver in proneural GBM. Consistently, CDK4/6 inhibition with palbociclib preferentially inhibited cell proliferation in vitro in a majority of proneural GSCs versus those of other subtypes. Palbociclib treatment significantly prolonged survival of mice with established intracranial xenografts of a proneural GSC line. We show that most of these sensitive PN GSCs expressed higher levels of CDK6 and had intact Rb1, while two GSC lines with CDK4 overexpression and null Rb1 were highly resistant to palbociclib. Importantly, palbociclib treatment of proneural GSCs upregulated mesenchymal-associated markers and downregulated proneural-associated markers, suggesting that CDK4/6 inhibition induced proneural-mesenchymal transition and underscoring the enhanced role of the E2F cell cycle pathway in the proneural subtype. Lastly, the combination of palbociclib and $\mathrm{N}, \mathrm{N}$-diethylaminobenzaldehyde, an inhibitor of the mesenchymal driver ALDH1A3, showed strong synergistic inhibitory effects against proneural GSC proliferation. Taken together, our results reveal that proneural GBM has increased vulnerability to CDK4/6 inhibition, and the proneural subtype undergoes dynamic reprogramming upon palbociclib treatment-suggesting the need for a combination therapeutic strategy.

\section{INTRODUCTION}

Glioblastoma (GBM) is the most common and most lethal primary brain tumor, causing $12-14,000$ deaths each year in the U.S. alone [1]. Median survival following diagnosis is approximately $12-15$ months with current therapy including maximal surgical resection, radiation, and temozolomide chemotherapy [2]. While all GBMs 
share histopathological and clinical features, The Cancer Genome Atlas (TCGA) and other profiling efforts have revealed two to four GBM subtypes: proneural (PN) and mesenchymal (MES) have been most reliably established, with classical (CL) and neural subtypes also described $[3,4]$. PN subtype typically arises in frontal cortex and often has PDGFRA amplification, IDH1/IDH2 mutation, and TP53 mutations; those with IDH1/IDH2 mutationwhich includes most secondary GBMs arising from lowgrade gliomas - have the best prognosis of any GBM subgroup, but proneural GBM without IDH mutations have perhaps the worst outcomes [3-5]. MES subtype is aggressive and has greater vascularity, and it has been associated with $N F 1$ lesions and with higher Akt, TGF- $\beta$, and NF- $\mathrm{KB}$ activity [3-5]. CL subtype is also aggressive and is marked by frequent $E G F R$ lesions [3-5]. The neural subtype has become controversial, as it is less distinct and may arise from substantial contamination of GBM samples with normal brain [5]. Major efforts have been underway to identify critical drivers of each GBM subtype, in hopes of gaining therapeutic leverage against them. Unfortunately, little progress has been made in uncovering key driver pathways and therapeutic vulnerabilities of the GBM subtypes, other than a few reports suggesting core circuitry of the MES subtype [6-8].

microRNAs (miRNAs) are endogenous 20-22 bp small RNAs that do not encode peptides, but powerfully regulate gene expression by blocking translation or impairing RNA stability of mRNAs with 3'-untranslated regions (3'-UTRs) containing target sites for those miRNAs [9]. Numerous miRNAs are dysregulated in cancer and play oncogenic or tumor-suppressive roles. The majority of over- and under-expressed miRNAs in cancer are likely dysregulated due to aberrant activity of oncogenic or tumor-suppressive pathways that regulate their expression. This suggests that signatures of dysregulated miRNAs may shed light on the core driver pathways within cancers.

To identify novel potential drivers in GBM subtypes, we performed in silico analysis of TCGA data and found that several in the miR-17 92 cluster or in analogous clusters are highly upregulated in the PN subtype of GBMs, and these are known to be transcriptionally up-regulated by the E2F cell cycle and myc pathways [10-12]. E2F drives cell cycle entry, and its activity is regulated by inputs from activators (CDK and cyclin proteins) and inhibitors (such as phosphorylated/activated $\mathrm{Rb} 1$ and the INK4 family $\mathrm{p} 15^{\mathrm{INK} 4 \mathrm{~b}} \mathrm{p} 16^{\mathrm{INK} 4 \mathrm{a}}, \mathrm{p} 18^{\mathrm{INK} 4 \mathrm{c}}$, and $\left.\mathrm{p} 19^{\mathrm{INK} 4 \mathrm{~d}}\right)$. The work published by TCGA has revealed that the CDK4/6-Rb-E2F pathway is frequently disrupted in $78 \%$ of GBMs. The most common alteration of this pathway is homozygous deletion of p15 and p16, which is present in $50 \%$ of tumors, as well as mutations in CDKN2A. Amplification/overexpression of CDK4 is detected in $15-20 \%$ of GBM, while homozygous deletion/ mutation of $R B 1$ is present in $7.6 \%$ of GBM. Amplification of CDK6 and homozygous deletion of p18 are less common $(2 \%)[5,13]$. Our TCGA microRNA findings, in addition to the frequent dysregulation of the E2F cell cycle pathway in GBM, led us to suspect the prominent involvement of this pathway in PN GBM and that it might represent a therapeutic vulnerability.

To further investigate whether the E2F cell cycle pathway was especially relevant in proneural GBM, we utilized palbociclib, a first-generation inhibitor of CDK4/6 recently approved by the FDA for breast cancer and now in clinical trials for patients with GBM $[14,15]$. In this study, we tested the relative sensitivity of a panel of GBM stem cell-like (GSC) lines to palbociclib, finding that it preferentially inhibits cell proliferation and induces G1 phase arrest in PN GSC lines with high expression of CDK6 and functional Rb1. Moreover, we show that CDK4/6 inhibition may induce PN-MES transition in GSCs, and that targeting both CDK4/6 and a MES driver synergistically inhibits proneural GSC proliferation.

\section{RESULTS}

\section{The miR-17 92 family and its paralogs are elevated in human PN GBM samples}

An early report on miRNAs in cancer indicated that miRNA expression patterns could better determine cancer tissue of origin than could gene expression arrays [19]. Similarly, we hypothesized that finding drivers of dysregulated miRNAs provides one approach to identifying core pathways for the GBM subtypes. As a first step toward identifying potential drivers of the GBM subtypes, we did in silico analysis of miRNA expression profiles using the TCGA GBM dataset and the previously identified subtyping of these samples [3]. We identified miRNAs with significantly dysregulated expression within each subtype versus others. As shown in Figure 1A and 1B, miR-219-5p, -577, -138, and -95 are down-regulated in all four subtypes compared to normal brain tissues, with the PN subtype exhibiting higher expression of each of these miRNAs. In contrast, miR-20a, -20b, -93, -106a, $-19 \mathrm{a},-130 \mathrm{~b}$, and $-10 \mathrm{~b}$ are up-regulated in GBM compared to the normal brain tissue. Notably, their expression is particularly elevated in PN GBM (Figure 1A, 1B and Supplementary Figure 1). Interestingly, miR-20a and -19a belong to the miR-17 92 family, while miR-20b and -106a belong to the miR-106a 363 family, a paralog of the miR-17 92 cluster [20]. The E2F family of transcription factors and c-myc can bind directly to the promoters of the miR-17 92 cluster and its paralogs to regulate their transcription $[10,11,21]$. Consistent with a greater role for E2F in PN GBM, E2F1 mRNA is significantly upregulated in the PN subtype versus the other subtypes (Figure 1C). Importantly, the expression levels of other major cell cycle-related genes such as CCND1, CCNB1, and $C C N B 2$ are also significantly elevated in the PN subtype 
compared to the other subtypes of GBM (Figure 1C and Supplementary Figure 2). Since the E2F pathway drives cell cycle progression and is regulated by CDK4 and CDK6, we postulated that CDK4/6 inhibition would be especially active against PN GBM.

\section{The miR-17 92 family and its paralogs are highly expressed in PN GSC lines}

We next assessed whether the miR-17-92 family and its paralogs are elevated in PN GSC lines. To test this, we utilized a panel of GBM neurosphere lines which were isolated from GBM patient samples and cultured in neural stem cell medium to maintain the original genetic features of the GBM patients [22]. PN and MES GBM are the most well-established subtypes, and may represent opposing ends of a phenotypic axis in GBM [22-24].
To investigate whether the GSC lines display these two distinct expression patterns, we performed qPCR to compare the expression of signature genes of the PN and MES subtypes. As shown in Figure $2 \mathrm{~A}$ and $2 \mathrm{~B}$, one group (8 GSC lines: G44, 448, 464, 559, 578, 806, 816 and 827) showed higher expression of what are considered PNassociated genes (SOX2, OLIG2, CD133 and NOTCH1) and the other (4 GSC lines: G34, 20, 22, and 267) showed higher expression of MES-associated genes (WT-1, LYN, TGFBR2 and BCL2A1). Since some MES markers (WT-1, $L Y N$ and $B C L 2 A 1)$ are relatively higher, but the PN markers (SOX2, OLIG2, CD133) are relative lower in the G528 line, we believe it belongs to the "other" or classical subtype. We then analyzed the expression of the miR17 92 cluster and a paralog in the 13 GSCs using qPCR. As predicted, the members of the miR-17 92 cluster (miR-20a, -20b, -93, -106a, -130b, and -10b) and paralog
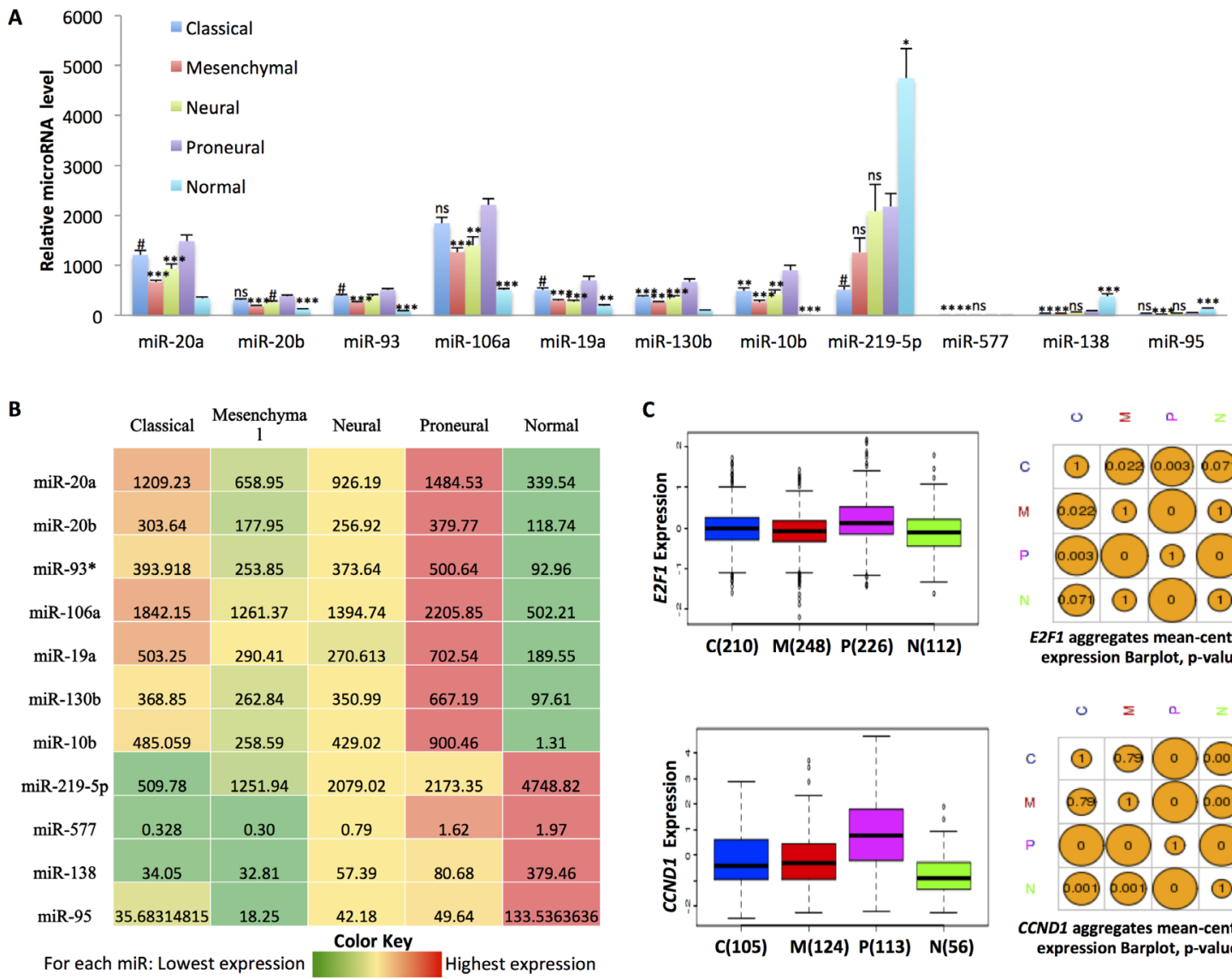

C
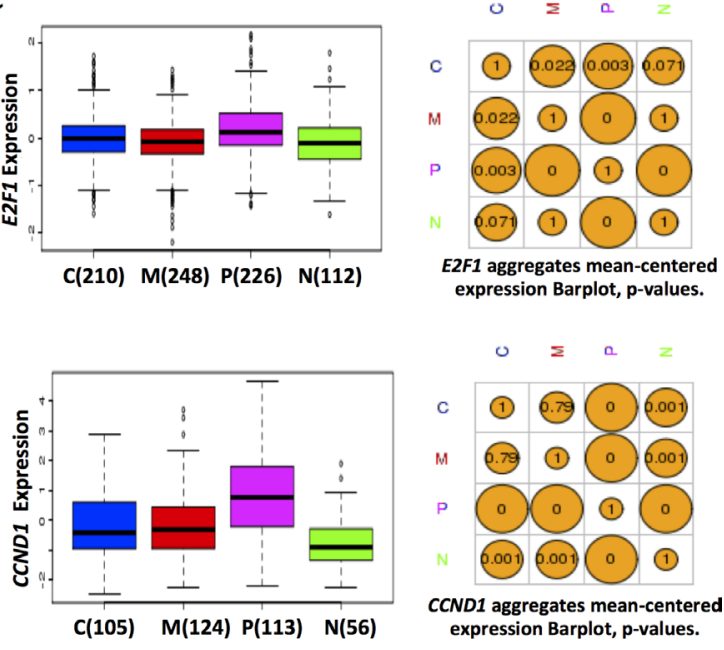

Figure 1: In silico analysis of the expression of selected miRNAs from the miR-17 92 cluster and paralogs shows elevated expression in proneural GBM. (A) Expression level of miR-17 92 and paralog miRNAs in the four subtypes of GBM versus normal brain tissues. Classical, $n=53$; Mesenchymal, $n=58$; Neural, $n=33$; Proneural, $n=57$; Normal, $n=10$. The difference was analyzed between proneural and the other three subtypes or normal brain tissues. ${ }^{*} p<0.05 ;{ }^{*} p<0.01 ;{ }^{*} p<0.001 ; * * * p<0.0001$; NS, not significant. Data are from TCGA and adapted from Project Betastasis: http://www.betastasis.com/glioma/tcga_gbm. (B) Heat map of the miR-17 92 family expression level in the four subtypes of GBM versus normal brain tissues based on the data shown in panel A. The number denotes the relative expression level of each miRNA in the four subtypes of GBM and normal brain tissues. (C) Expression of E2F1 and CCND1 in the four subtypes of GBM. C: classical; M: mesenchymal; P: proneural; N: neural. The sample number is shown in the left panel. The $p$ values comparing across subtypes are shown in the right panel. Data are adapted from the Glioblastoma Bio Discovery Portal: https://gbm-biodp.nci.nih.gov. 
clusters were over-expressed in seven of eight PN GSC lines (G44, 448, 464, 559, 578, 816 and 827; Figure 2C).

\section{Most PN GSC lines show greater sensitivity to CDK4/6 inhibitors}

Given that the E2F cell cycle pathway might play a greater role in PN GBM, we then subjected a panel of twelve GSC lines and a normal human astrocyte (NHA) line to treatment with the CDK4/6 inhibitor palbociclib.
The cells were treated with varying concentrations of palbociclib or vehicle for five days, and then assayed for cell number by the CyQUANT Direct Cell Proliferation assay (which measures cell number based on both DNA content and membrane integrity, but is independent of the metabolic state of cells) (Figure 3A). IC50 values for individual cell lines were also determined (Table 1). NHA are highly resistant to palbociclib, with an IC50 of greater than $5 \mu \mathrm{M}$. All four MES lines were relatively resistant to the CDK4/6 inhibitor, and the IC50s are equal to or greater

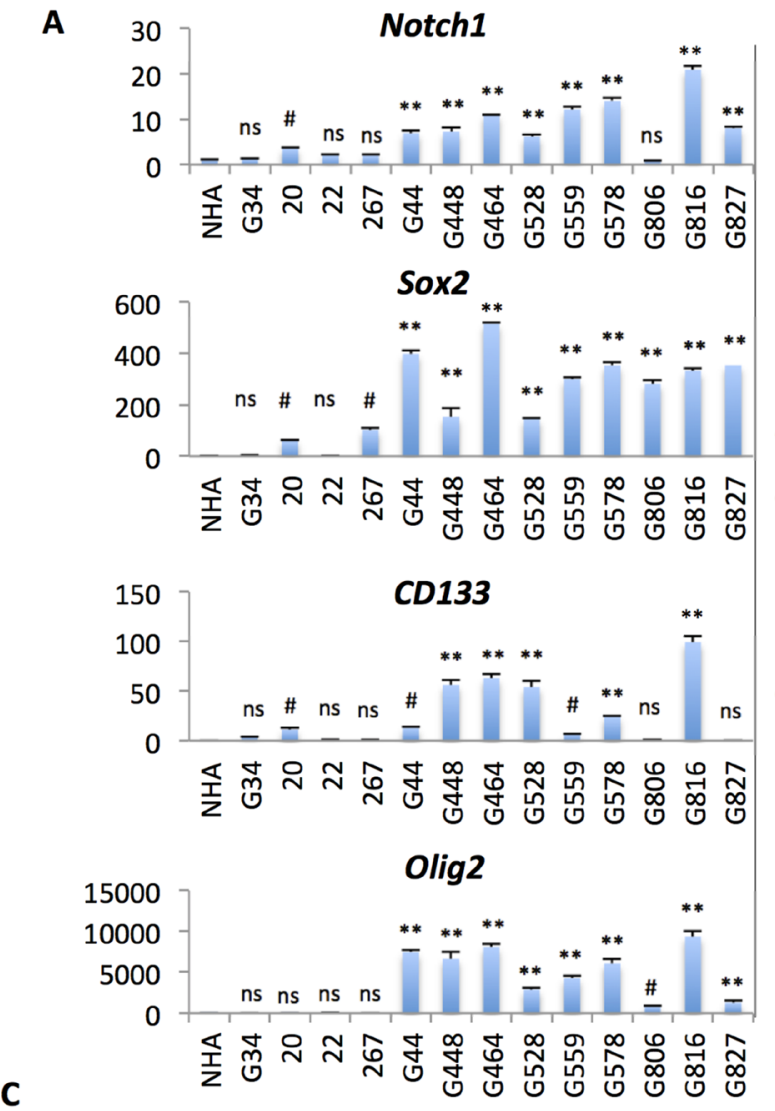

B
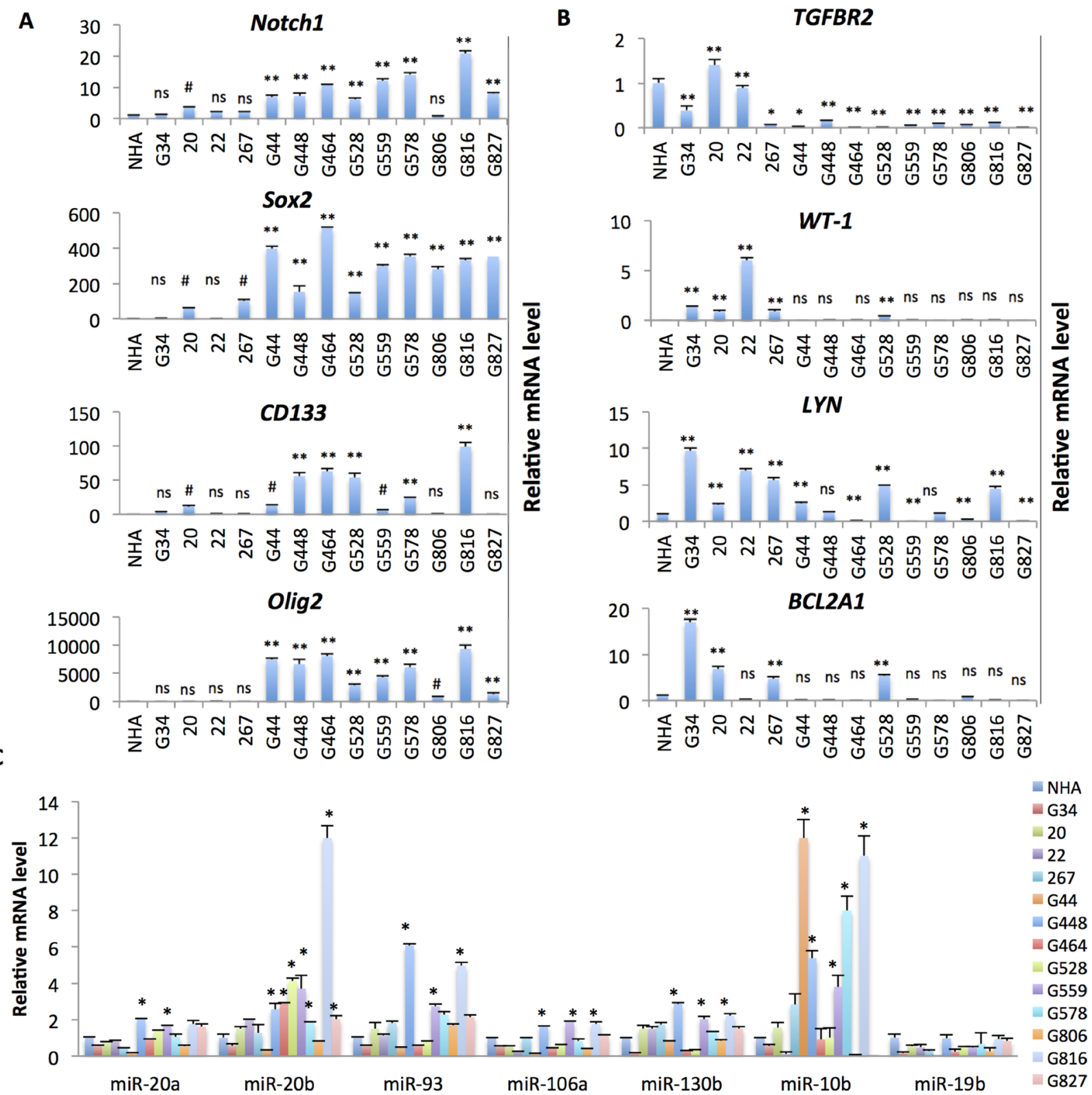

Figure 2: Expression of PN markers and the miR-17 92 family is elevated in a set of PN GSC lines. (A and B) RT-qPCR analysis of the markers for PN GBM (A, OLIG22, SOX2, CD133 and NOTCH1) and MES GBM (B, TGFBR2, WT-1, LYN and BCL2A1) in the GSC lines and normal human astrocytes (NHA). $n=3$. NS, not statistically significant; ${ }^{*} p<0.05 ;{ }^{*} p<0.01 ; * * p<0.001$; as compared to the expression level in NHA. (C) qPCR analysis of the expression of miR-17 92 family miRNAs and paralogs in the GSC lines and NHA. $n=3 .{ }^{*} p<0.01$, as compared to the expression level in NHA. Shown are representative data of three independent experiments with similar results. 
Table 1: IC50s and molecular features of the GSCs

\begin{tabular}{|c|c|c|c|c|c|c|c|c|c|}
\hline weGSC & Subtype & IC50(nM) & CDKN2A(p16) & CDKN2B(p15) & CDKN2C(p18) & CDKN2D(p19) & CDK4 & CDK6 & RB1 \\
\hline G34 & MES & 1000 & - & - & + & + & + & + & + \\
\hline 20 & MES & 5000 & + & + & + & + & + & - & - \\
\hline 22 & MES & 1000 & - & + & + & + & + & + & - \\
\hline 267 & MES & $>5000$ & - & + & + & + & + & + & + \\
\hline G44 & $\mathrm{PN}$ & 210 & - & - & + & + & + & ++ & + \\
\hline G448 & $\mathrm{PN}$ & 100 & - & - & + & + & + & ++ & + \\
\hline G464\# & $\mathrm{PN}$ & 4500 & + & + & + & + & +++ & + & - \\
\hline G528\# & Other & $>5000$ & + & - & + & + & +++ & - & - \\
\hline G559 & PN & 100 & - & - & + & + & + & +++ & + \\
\hline G578 & PN & 50 & + & - & + & + & + & ++ & + \\
\hline G816 & $\mathrm{PN}$ & 80 & - & - & + & + & + & +++ & + \\
\hline G827 & PN & 9 & - & - & + & + & + & + & - \\
\hline
\end{tabular}

The IC50 of palbociclib is based on the cell survival results shown on Figure 3A. \# indicates the two resistant lines; -, negative expression; +, positive expression. Number of + indicates relative expression level.

than $1 \mu \mathrm{M}$. In contrast, six of seven PN GSC lines (G44, $448,559,578,816$ and 827 ) were extremely sensitive to the inhibitors, with IC50s less than $210 \mathrm{nM}$. Interestingly, one PN line (G464) and one other subtype line (G528) showed stronger drug resistance, exhibiting IC50 values greater than $4.5 \mu \mathrm{M}$ (Figure 3A, Table 1). Similar doseresponse curves were obtained from these PN lines and G528 treated with LEE001, a second CDK4/6 inhibitor (Supplementary Figure 3).

The impact of palbociclib treatment on cell cycle distribution was also analyzed using flow cytometry. In the most sensitive PN lines, palbociclib induced accumulation of cells in G1 phase (Figure 3B). In contrast, CDK4/6 inhibition failed to induce $\mathrm{G} 1$ arrest in the three resistant lines, NHA, G464, or G528 (Figure 3B). Moreover, daily oral palbociclib treatment $(150 \mathrm{mg} / \mathrm{kg})$ significantly increased survival in mice with established intracranial xenografts of the PN GSC line G448, one of the few PN lines showing tumorigenicity in vivo (Figure $3 \mathrm{C}$ ). Taken together, GSC lines exhibited differential sensitivity to the CDK4/6 inhibitor, with most PN GSCs more sensitive to palbociclib than GSCs of other subtypes.

\section{Molecular abnormalities in the E2F cell cycle pathway in GSC lines}

We next explored the mechanisms underlying the differential response to CDK4/6 inhibition across the GSC lines. To this end, we investigated selected components of the E2F cell cycle pathway in the twelve GSC lines using qPCR and immunoblot. Of the six sensitive PN GSC lines, five expressed higher levels of CDK6, whereas all four MES lines expressed lower levels of CDK6. Interestingly, the two most resistant lines (G464 and G528) displayed elevated expression of CDK4 (Figure 4A). Since Rb is tightly regulated by CDK4 and 6 and their cyclin D binding partners, as well as the inactivating CDK inhibitors such as $\mathrm{CDKN} 2 \mathrm{~A}\left(\mathrm{p} 16^{\mathrm{INK} 4 \mathrm{~A}}\right), \quad \mathrm{CDKN} 2 \mathrm{~B}\left(\mathrm{p} 15^{\mathrm{INK} 4 \mathrm{~B}}\right)$, $\operatorname{CDKN} 2 \mathrm{C}\left(\mathrm{p} 18^{\mathrm{INK} 4 \mathrm{C}}\right)$, and $\mathrm{CDKN} 2 \mathrm{D}\left(\mathrm{p} 19^{\text {INK4D }}\right)$, we also examined the expression pattern of the four CDK inhibitors. Expression of $p 16^{I N K 4 A}$ was only detected in four of twelve GSC lines (20, G464, G528 and G578), and $p 15^{I N K 4 B}$ was present only in $20,22,267$ and G464 cells. In contrast, $p 18^{I N K 4 C}$ and $p 19^{I N K 4 D}$ are expressed in all the GSC lines (Figure 4B). Rb1 is present in five of the six sensitive PN GSC lines, with G827 the exception. Of note, $\mathrm{Rb} 1$ protein appears absent in the two most resistant GSC lines, G464 and G528. Rb1 protein was present in some of the resistant MES lines, such as G34, 20 and 267 (Figure 4A), indicating that factors other than Rb1 status may determine palbociclib response in some GSC linesadding to the primary mechanism of palbociclib resistance reported previously [25-27]. The molecular features of the GSC lines are summarized in Table 1. In summary, it appears that the sensitive PN lines express higher levels of CDK6 with intact Rb1, and the resistant PN lines express higher level of CDK4 without Rb1.

\section{Palbociclib decreases Rb1 phosphorylation and reduces miR-17 92 family and paralog expression in the sensitive PN GSC lines}

CDK4/6-Cyclin D-mediated Rb phosphorylation drives cell cycle progression by releasing E2F transcription factors [28]. Therefore, immunoblot was conducted to examine the effects of palbociclib on Rb1, E2F1, and Cyclin D1 protein expression. In five of the six sensitive PN lines (G44, 448, 559, 578, and 816), palbociclib treatment for 1 day or 5 days dramatically suppressed total Rb1 protein as well as phospho-Rb1 (Ser 807/811) levels. Expression of E2F1, the key target of $\mathrm{Rb}$, was also reduced upon palbociclib administration (Figure 5A and Supplementary Figure 2). Cyclin D1 level was increased in G44, 559, and 578 lines, which is in line with previous reports in other 
cell types [31-34]. In contrast, in the Rb1-deficient PN line, G827, no obvious changes in the expression levels of E2F1 and Cyclin D1 were detected upon palbociclib treatment (Figure 5A, Supplementary Figure 4).

As described earlier, E2F1 drives expression of the miR-17 92 family and its paralogs, which are highly expressed in PN GSCs. We examined if palbociclib is able to affect expression of these miRNAs using qPCR. As expected, the CDK4/6 inhibitor significantly decreased the expression of miRNAs in these clusters, including miR20a, $-20 \mathrm{~b},-93^{*}$, and $-106 \mathrm{a}$ in proneural GSCs (G44 and
G559; Figure 5B). Interestingly, palbociclib also reduced expression of two other miRNAs elevated in PN GBM, miR-130b (in both G44 and G559 PN GSC lines) and miR-10b (in G559 PN GSC line).

\section{Palbociclib may induce a proneural- mesenchymal transition that can be addressed with addition of a MES-selective agent}

Palbociclib has been shown to be involved in an Epithelial-Mesenchymal transition (EMT) in pancreatic
A
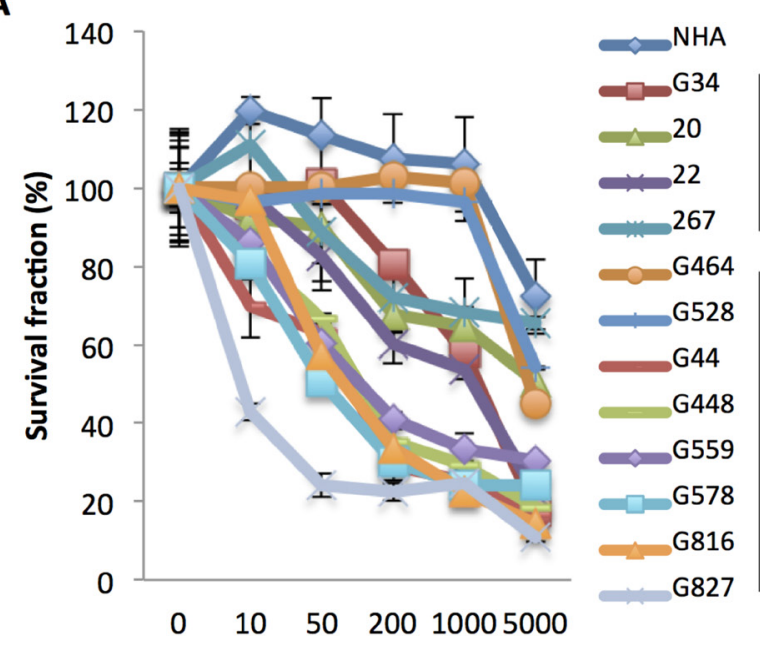

Concentration of pabociclib (nM)

C

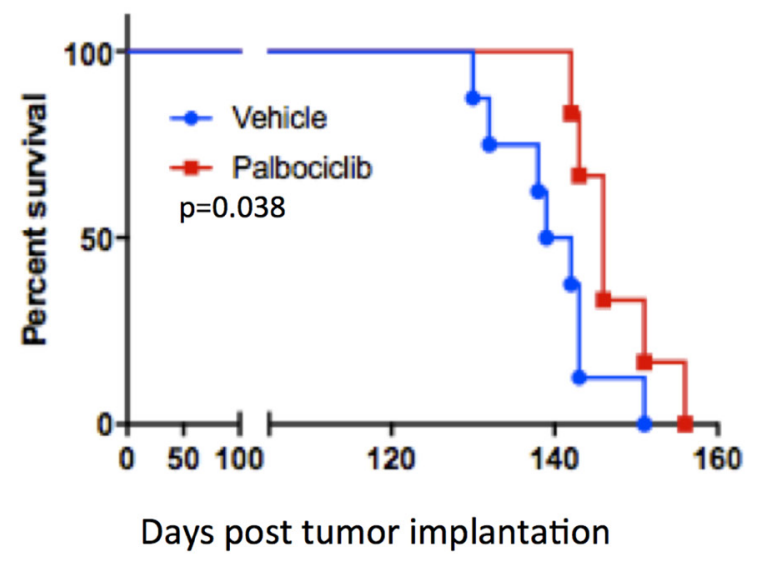

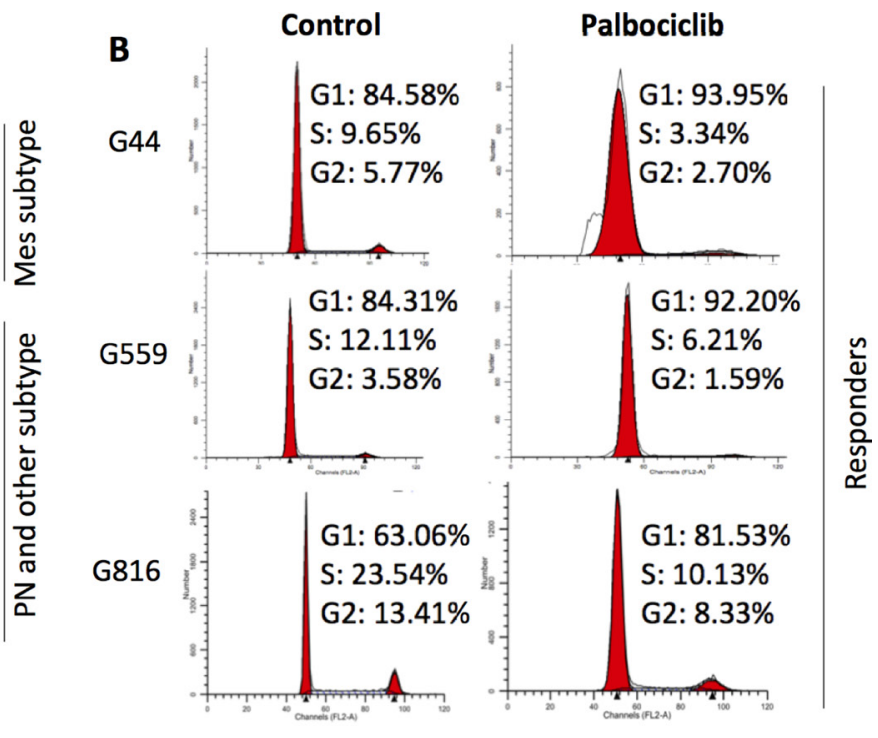

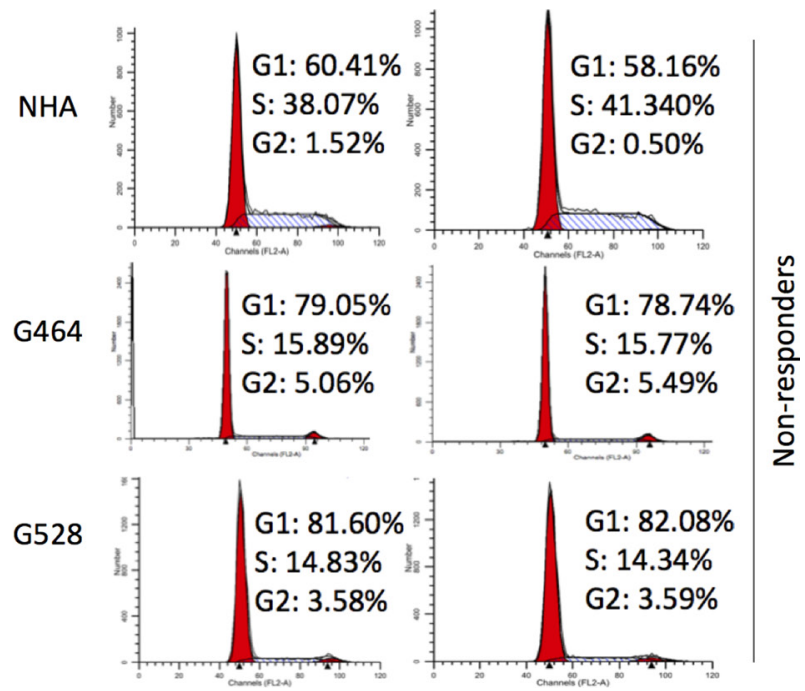

Figure 3: PN GSCs are more sensitive to palbociclib than other GSC subtypes. (A) Effect of palbociclib on cell proliferation. The twelve GSC lines and normal human astrocytes were seeded at a density of 3,000 cells/well on laminin $(10 \mu \mathrm{g} / \mathrm{ml} \mathrm{in} 0.01 \% \mathrm{poly}-$ ornithine) coated 96-well plates and treated with the indicated doses of palbociclib for 5 days. $n=3$. Relative cell number was determined by CyQUANT Direct Cell Proliferation assay. (B) Effect of palbociclib on GSC cell cycle distribution. Responder and non-responder lines are shown in left and right panel, respectively. The cells were treated with palbociclib $(1 \mu \mathrm{M})$ for 5 days prior to the flow cytometry analysis. Shown are representative data of three independent experiments with similar results. (C) Kaplan-Meier survival curves of mice after intracranial implantation of G448 $\left(5.0 \times 10^{5}\right.$ cells/mouse $)$ treated with vehicle or palbociclib $(150 \mathrm{mg} / \mathrm{kg} / \mathrm{day}, 8 \mathrm{mice} / \mathrm{group})$. The treatment started on day 14 post inoculation and lasted for 4.5 months. Statistical comparisons were performed using Gehan-Breslow-Wilcoxon test. $p=0.038$. 
and breast cancer $[29,33]$. Given these findings in other cancers and our demonstration of proneural sensitivity to palbociclib, we hypothesized that CDK4/6 inhibition might trigger a shift in GSC subtype. We performed RT-qPCR and found that palbociclib treatment of PN GSC lines decreased expression of 4 PN markers (NOTCH1, OLIG2, $C D 133$ and $S O X 2$ ), while expression of the MES markers TGFBR2, BCL2A1, and WT-1 increased (Figure 6A) - suggesting a possible PN-MES transition (PMT) in palbociclib-treated PN GSCs.

Since ALDH1A3 has been recently shown to be aberrantly up-regulated in MES GSCs compared with PN GSCs [22], we examined whether its expression is affected by CDK4/6 inhibition. A low dose of palbociclib (10 nM) significantly up-regulated $A L D H 1 A 3$ mRNA expression in PN GSC lines ( $p<0.01$, Figure 6B). We then examined if an ALDH1A3 inhibitor, DEAB, might be able to oppose the prospective PN-MES transition and act synergistically with palbociclib. DEAB $(25 \mu \mathrm{M})$ alone had no obvious effect on the proliferation of PN GSC lines. However, when it was combined with palbociclib $(10 \mathrm{nM})$ to treat PN GSCs, cell proliferation was significantly inhibited compared to the cells treated with palbociclib or DEAB alone (For G44, CDI $=0.79$; for $\mathrm{G} 559, \mathrm{CDI}=0.565$; for G816, CDI =0.677; Figure 6C). Taken together, these data suggest that palbociclib treatment of PN GSCs induces a possible PN-MES transition that can potentially be ameliorated with the addition of an agent with reported MES-selective activity.

\section{DISCUSSION}

While previous reports have linked certain microRNA profiles to particular GBM subtypes [34-36], to our knowledge this is the first example of leveraging subtype-overexpressed miRNAs to identify underlying driver pathways. While studies of gene expression have been the focus of past studies to identify upstream core transcriptional drivers of subtypes in GBM and other cancers, the far smaller number of miRNAs [37] may facilitate such efforts. Here, our in silico analysis showed the miR-17 92 cluster and its paralogs to be elevated in PN GBM, potentially indicating an enhanced role for the E2F cell cycle pathway and potential sensitivity to a CDK4/6 inhibitor in PN GBM. Hints in prior reports also supported this hypothesis. One report demonstrated that the miR-17 92 cluster targets TGF- $\beta$ signaling, a potential driver of mesenchymal GBM, and therefore over-expression of this miRNA cluster should push cancer cells toward a proneural or epithelial phenotype [38]. A more recent study showed that platelet-derived growth factor (PDGF), known to play a significant role in proneural GBM, drives E2F-USP1 signaling in proneural glioma [39].

It has been reported that $\mathrm{Rb}$ status is the primary determinant of sensitivity to CDK4/6 inhibition [40]. In breast cancer, $\mathrm{Rb} 1$ deficiency or loss of its function results in palbociclib resistance, and the same has been shown for GBM [30, 40-45]. In line with these results, one report has shown that changes in the E2F-Rb1 cell cycle pathway, including higher levels of Cyclin D1 and Rb1 and lower level of p16, led to the greatest sensitivity to palbociclib treatment [46]. Another report has shown that co-deletion of $p 16$ and $p 18$ predicts palbociclib sensitivity, and that higher levels of $C D K 4$ or $C D K 6$ had no influence on sensitivity [44]. However, this was not the case in our study, in which we found p18 to be present in all 12 GSC lines and p16 to be detectable in the two most resistant lines (G464 and G528). In contrast, in line with a recent report [45], our results suggest that $\mathrm{CDK} 4$ overexpression may be a marker for palbociclib resistance even in proneural GBM, while CDK6 over-expression may be associated with sensitivity. Specifically, nearly all of the sensitive PN GSC lines express higher level of CDK6 and have $\mathrm{Rb} 1$ protein expression, while p15 and p16 are absent in these cells.

It has been noted that palbociclib may also have an Rb1-independent anti-proliferative effect. Daniel et al. recently observed similar responses to palbociclib in $R b 1$-wild-type and $R b 1$-mutant bladder cell lines in vitro
A

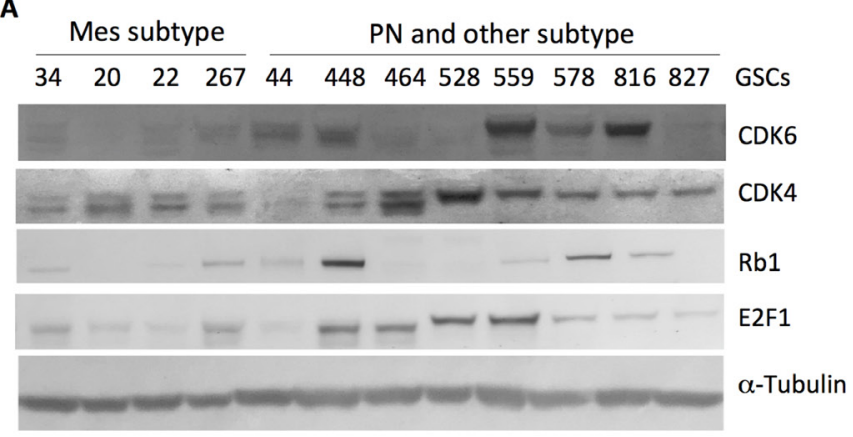

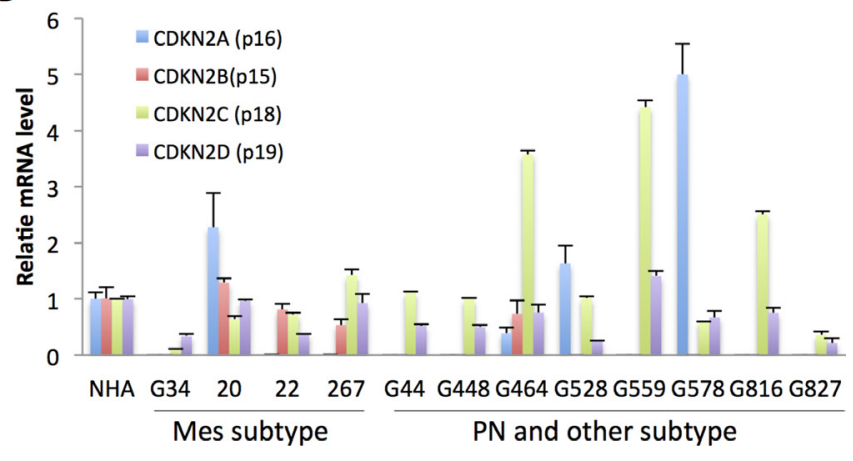

Figure 4: Molecular features of the GSC lines suggest associations with sensitivity and resistance. (A) Immunoblot analysis of CDK4, CDK6, Rb1, and E2F1 in the GSC lines. $\alpha$-Tubulin was used as a loading control. (B) qPCR analysis of $p 15, p 16, p 18$, and $p 19$ expression levels in the GSC lines and NHA. 
and in xenografts in vivo [25]. Similarly, palbociclib has proven activity in Rb1-deficient prostate cancer cells [26] and hepatocellular carcinoma cells in vitro, in which some activity in Rb1-deficient cells may be compensated by related proteins such as p107 [27]. Interestingly, in our study an Rb1-deficient PN GSC line (G827) displayed the greatest sensitivity to palbociclib treatment. While our study and others suggest additional markers for palbociclib sensitivity, further work on this important area is needed.

Inhibition of $\mathrm{Rb} 1$ phosphorylation is a downstream mediator of CDK4/6 inhibition. We showed that palbociclib decreased total Rb1 levels as well as Rb1 phosphorylation, likely due to enhanced Rb1 degradation. We also observed that palbociclib increased Cyclin D1 levels in half of the sensitive GSC lines. As has been suggested by Dean et al. [30], Cyclin D1 stability is likely increased through proximal effects on the CDK4/6-Cyclin D1 complex, and the elevated Cyclin D1 could fuel rapid activation of CDK4/6 if the levels of palbociclib were to become limiting-suggesting that continual treatment might be needed. Notably, elevated Cyclin D1 is implicated in both EMT and cell senescence [31-32, 47]. Interestingly, CDK4/6 inhibition also decreased E2F1 expression in the Rb1-intact sensitive GSCs, but had no obvious effect on E2F1 protein expression in the Rb1deficient line G827. Since it had a similar anti-growth effect in these lines, this may indicate that other effectors are also involved in this process.

These results indicated that CDK4/6 inhibition in PN GBM decreased expression of PN markers and increased MES marker expression, indicating there might be a shift from PN to MES subtype. A similar phenomenon has been observed in pancreatic cancer models, in which palbociclib treatment prompted an EMT response [29]. However, Qin et al. reported that palbociclib inhibits EMT in breast cancer [33], indicating that EMT induced by CDK4/6 inhibition might be cell type-dependent. While a treatment-induced PN-MES shift in GBM has been

A
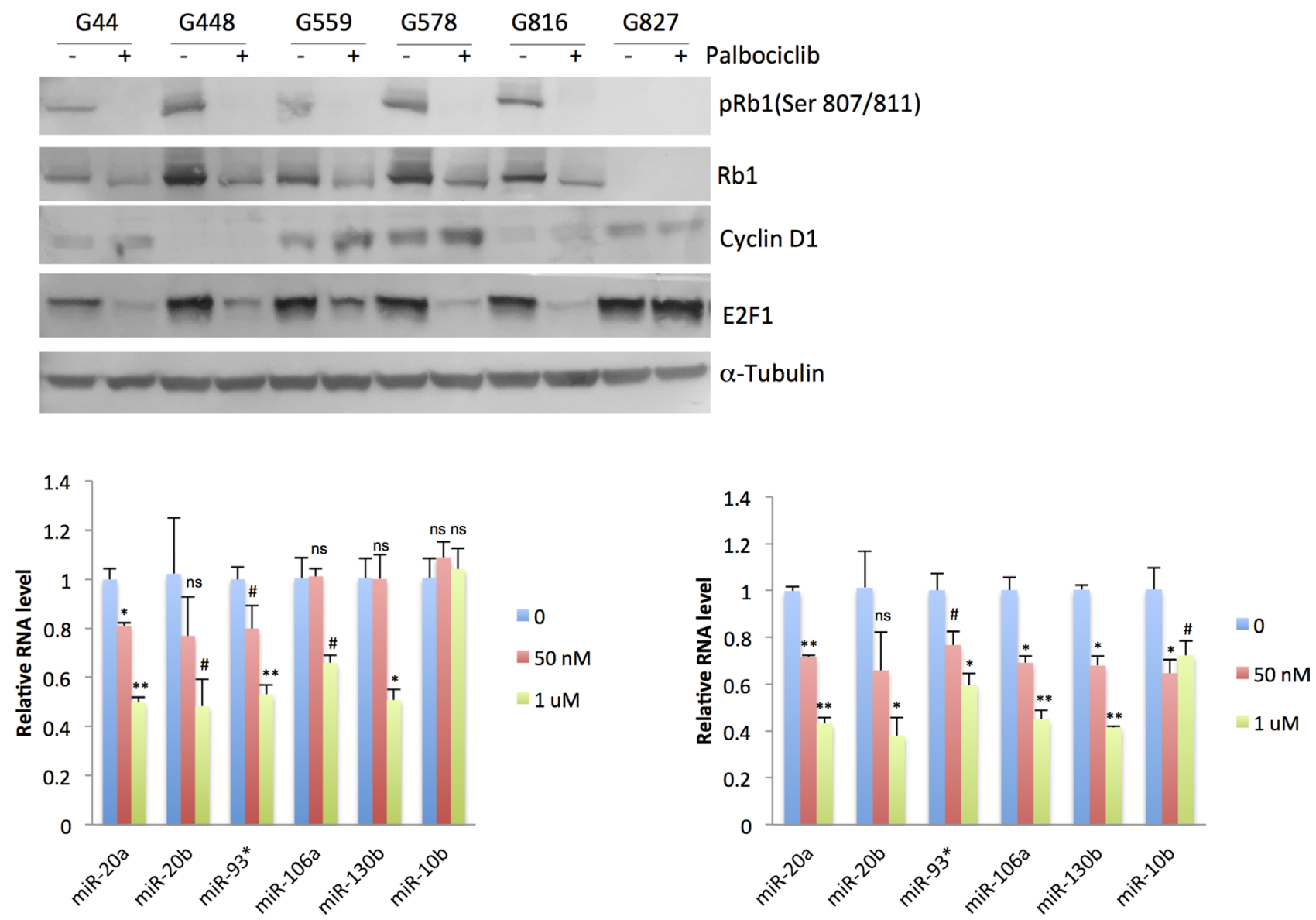

Figure 5: Palbociclib reduces Rb1 phosphorylation and miR17 92 and paralog expression in the responder PN GSCs. (A) Immunoblot analysis of Rb1, phospho-Rb1 (Ser 807/811), Cyclin D1, and E2F1 in the palbociclib (1 $\mu$ M, $24 \mathrm{hr})$-treated GSCs. $\alpha$-Tubulin was used as loading control. (B) qPCR analysis of miR-17 92 and paralog expression in G44 (left panel) and G559 (right panel) treated with the indicated doses of palbociclib for 5 days. $n=3$. Differences were analyzed between the control and the palbociclib-treated groups. NS: not statistically significant; ${ }^{*} p<0.05 ;{ }^{*} p<0.01:{ }^{*} p<0.001$. Shown are representative data of three independent experiments with similar results. 
proposed in other reports $[3,48]$, the rapidity of it in these experiments was remarkable. This prompted us to seek an answer to what seems to be an acute resistance mechanism. We found that a low concentration of palbociclib was able to induce the expression of ALDH1A3, previously shown to be a MES marker and potential driver in GBM, and which plays a role in the initiation and progression of tumors via the clearance of aldehydes and the production of retinoic acid [22]. Importantly, combination of the ALDH1A3 inhibitor, DEAB, with palbociclib synergistically inhibited proneural GSC cell proliferation.
In summary, we show that miRNA expression profile analysis may provide new insights into the druggable drivers of cancer subtypes such as PN GBM. CDK4/6 inhibition has an enhanced anti-proliferative effect against most PN GSCs, and a rapid PN-MES transition may occur as a protective mechanism. We propose that palbociclib may best be applied against PNpredominant GBM, and that combination with a MESselective agent such as DEAB may help block resistance. These findings may also have relevance for other cancers, given that the proneural-mesenchymal axis in GBM
A

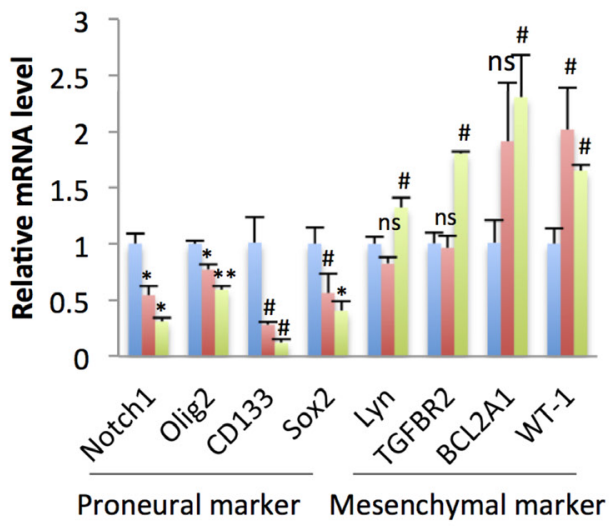

B

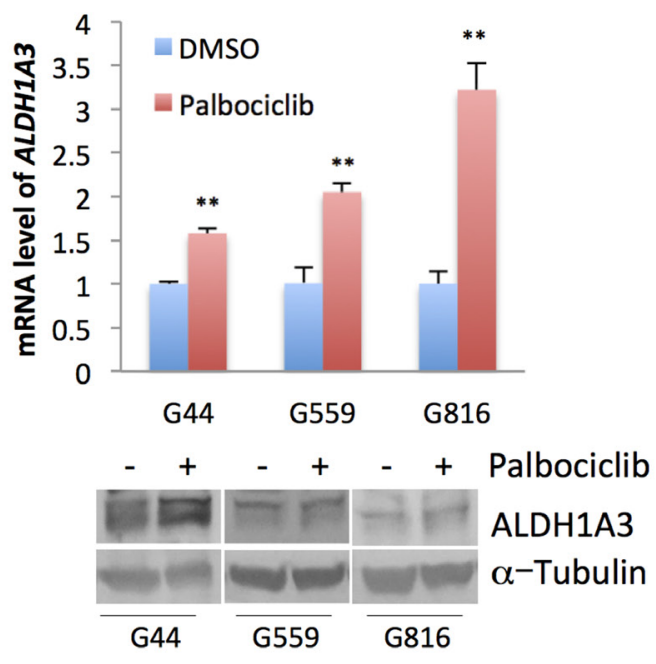

DMSO

$50 \mathrm{nM}$

$1 \mathrm{uM}$
G559

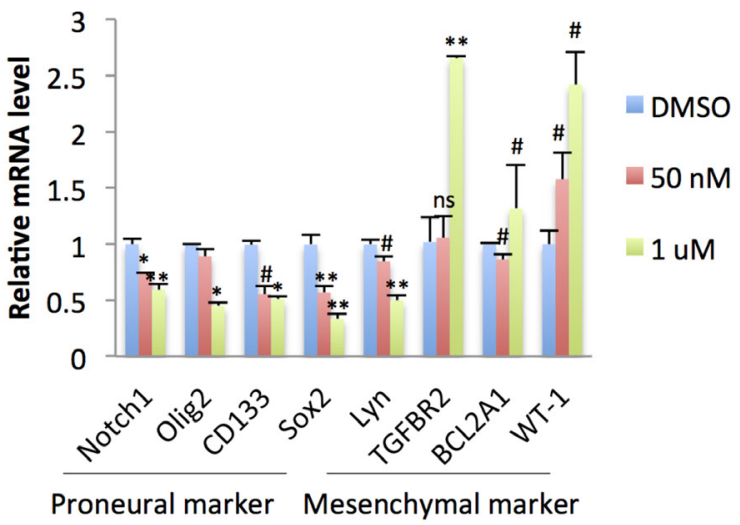

C

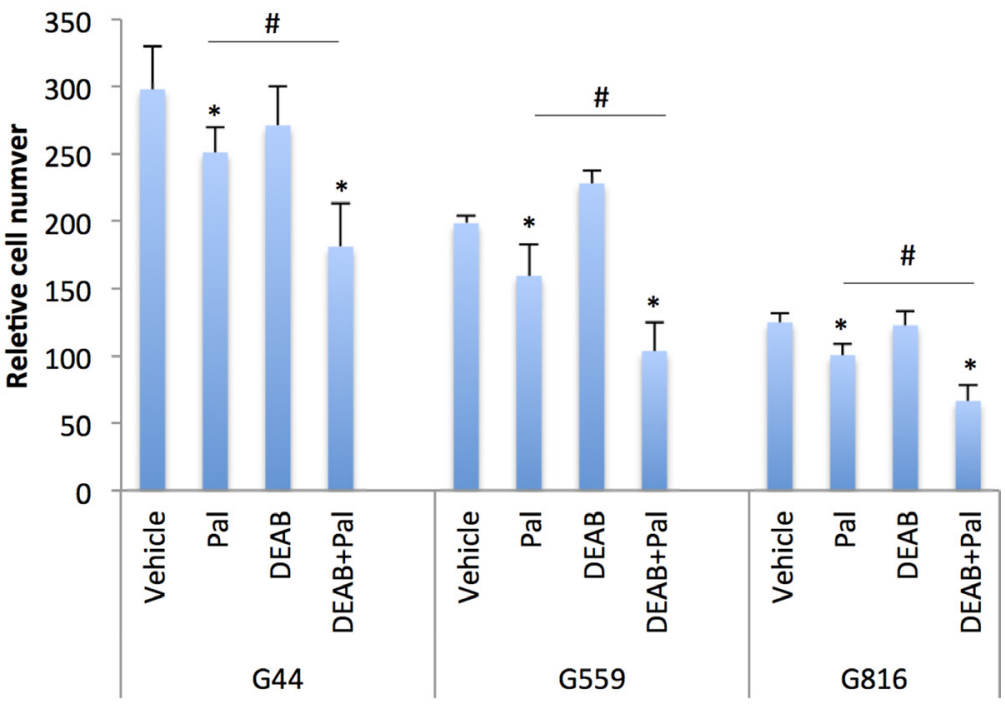

Figure 6: Palbociclib induces a potential transition of PN GSCs to MES GSCs. (A) qPCR analysis of markers of PN and MES subtypes in G44 and G559 treated with the indicated doses of palbociclib for 5 days. $n=3$. Differences were analyzed between the control and the palbociclib-treated groups. NS: not statistically significant; $\# p<0.05 ; * p<0.01 ; * * p<0.001$. (B) qPCR and immunoblot analysis of ALDH1 A3 in three independent PN GSC lines that were treated with $10 \mathrm{nM}$ of palbociclib for 5 days. $n=3$. Differences were analyzed between the control and the palbociclib-treated groups. NS: not statistically significant; ${ }^{*} p<0.05 ;{ }^{*} p<0.01 ; * * p<0.001$. (C) Cell proliferation analysis of three independent PN GSC lines that were treated with palbociclib $(10 \mathrm{nM})$, DEAB $(25 \mu \mathrm{M})$, or both for 5 days. The cells were cultured on a laminin $(10 \mu \mathrm{g} / \mathrm{ml}$ in poly ornithine)-coated 96-well plate. Cell number was determined by CyQUANT Direct Cell Proliferation assay. $n=3 .{ }^{*} p<0.05$, comparison between palbociclib or DEAB treatment with the vehicle control; ${ }^{*} p<0.05$, comparison between combination group with palbociclib treatment. G44, CDI $=0.79$; G559, CDI $=0.565 ; \mathrm{G} 816, \mathrm{CDI}=0.677$. Shown are representative data of three independent experiments with similar results. 
seems to parallel the epithelial-mesenchymal axis in many epithelial malignancies.

\section{MATERIALS AND METHODS}

\section{Cell culture and reagents}

GSC lines were obtained from Jeongwu Lee (Cleveland Clinic), Jakub Godlewski and Agnieszka Bronisz at Brigham and Women's Hospital, Ichiro Nakano (University of Alabama), and Krishna Bhat and Erik Sulman (MD Anderson Cancer Center) and were derived as previously described [6]. The GSC lines were cultured in Neurobasal medium supplemented with N-2 (Invitrogen), B27 (Invitrogen), glutamine, EGF (25 ng/ml) and bFGF $(25 \mathrm{ng} / \mathrm{ml})$ to maintain the stemness and genetic features of the clinical sample. When necessary, the GSC lines were cultured in the above stem cell medium on tissue culture plates coated with laminin (Roche, $10 \mu \mathrm{g} / \mathrm{m}$ in $0.01 \%$ poly-ornithine, Sigma). This laminin monolayer culture condition was previously reported to be equivalent to neurosphere culture for glioblastoma stem cell-like lines $[16,17]$. Human origin of GBM stem cell lines was confirmed by short tandem repeat analysis within the last six months.

Immortalized normal human astrocytes (NHA) were maintained in DMEM with 10\% FBS. Palbociclib and LEE001 (ribociclib) were purchased from Selleckchem. $\mathrm{N}, \mathrm{N}$-diethylaminobenzaldehyde (DEAB) was obtained from Sigma.

\section{In silico gene and miRNA expression analysis}

The expression profiles of miRNAs and genes were analyzed using the online TCGA GBM dataset: 1) Project Betastasis: http://www.betastasis.com/glioma/tcga_gbm; 2) The Glioblastoma Bio Discovery Portal: https://gbmbiodp.nci.nih.gov.

\section{Western blots and antibodies}

Cells were lysed in RIPA buffer (Cell Signaling Technology) with complete protease and phosphatase inhibitor (Roche). Primary antibodies used for Western blot were: Rb1 (9309), phopho-Rb1 (Ser807/811) (9308), Cyclin D1 (2922), CDK6 (3136), CDK4 (12790), and E2F1 (3742) (Cell Signaling Technology), ALDH1A3 (NBP2-15339, Novus Biologicals), and $\alpha$-Tubulin (Sigma). Secondary anti-Rabbit IgG and anti-mouse IgG were purchased from Sigma.

\section{Cell proliferation assay}

Cell viability was assessed using the CyQUANT Direct Cell Proliferation Kit (ThermoFisher Scientific). Cells were plated in triplicate onto a laminin $(10 \mu \mathrm{g} / \mathrm{ml}$ in $0.01 \%$ poly-ornithine) coated 96 -well plate at a concentration of 3,000 cells/well and began treatment the next day with palbociclib or vehicle for 5 days. The detection reagent was added to wells at a 1:1 ratio and incubated at $37^{\circ} \mathrm{C}$ for $1 \mathrm{hr}$. Fluorescence was measured at 480/535 nm using GENios Pro (Tecan).

\section{Flow cytometry analysis}

Cell cycle distribution was analyzed on $\mathrm{BD}$ Facscalibur at the Flow Core of the University of Virginia. The palbociclib-treated cells were collected, washed with PBS, and fixed in ice-cold $70 \%$ ethanol $/ 10 \%$ PBS for at least $2 \mathrm{hr}$. The fixed cells were then centrifuged for $5 \mathrm{~min}$ at $300 \mathrm{~g}$, resuspended in $1 \mathrm{ml}$ propidium iodide (PI) staining solution with $100 \mu \mathrm{g} / \mathrm{ml}$ RNase A and $0.05 \%$ Triton X-100, and incubated for $30 \mathrm{~min}$ at $37^{\circ} \mathrm{C}$. The results were analyzed using Modfit software.

\section{qPCR}

Total RNA was harvested by QIAzol reagent (Qiagen) and reverse-transcribed (SuperScript III First Strand kit; Invitrogen). For gene expression analysis, qPCR was performed with $2 \mu 1$ of diluted cDNA on an Applied Biosystems StepOnePlus PCR machine using Power SYBR Green (Applied Biosystems) according to the manufacturer's instructions. All reactions were performed in triplicate and repeated at least two times. Relative quantification was performed for each sample and normalized with GAPDH expression for comparison. Primers used for qPCR were listed in Supplementary Table 1. Gene expression levels were calculated using the relative $\Delta C \mathrm{t}$ method.

For miRNA expression analysis, single-stranded cDNAs from total RNA samples were synthesized using the TaqMan MicroRNA Reverse Transcription Kit (Applied Biosystems). Mature miRNA expression was determined using small RNA TaqMan assays according to the manufacturer's instructions (Applied Biosystems). All reactions were done in triplicate. The expression of miRNAs was normalized using RNU48. The expression relative to RNU48 was determined using the $\Delta C \mathrm{t}$ method.

\section{Xenograft model}

All animal experiments conformed to ethical principles and guidelines approved by the Institutional Animal Care and Use Committee at the University of Virginia. $5.0 \times 10^{5}$ of G448 cells in $5 \mu \mathrm{l}$ PBS were injected intracranially into 6-8 week old SCID Ncr mice. Beginning 14 days after surgery, $100 \mu \mathrm{l}$ of palbociclib $(150 \mathrm{mg} / \mathrm{Kg}$ in water) or water was given daily via oral gavage (8 mice/ group) [37].The survival and health of the tumor-bearing mice were closely monitored. Survival data were plotted on a Kaplan-Meier curve. Statistical comparisons were performed using Gehan-Breslow-Wilcoxon test. 


\section{Statistical analysis}

Data are expressed as the mean $\pm \mathrm{SD}$. Statistical analyses were performed using GraphPad Prism 7.00. Student's $t$ test was used to compare the difference between the control and the treatment group. One-way ANOVA was used to compare the difference between multiple groups. $P<0.05$ was considered statistically significant.

The coefficient of drug interaction (CDI) was used to analyze drug combinatorial effects [18]. CDI is calculated as follows: $\mathrm{CDI}=\mathrm{AB} /(\mathrm{A} \times \mathrm{B})$. $\mathrm{AB}$ is the ratio of the combination groups to the control group; $\mathrm{A}$ or $\mathrm{B}$ is the ratio of the single agent groups to the control group. $\mathrm{CDI}<1$ indicates synergism, and $\mathrm{CDI}<0.7$ indicates a significantly synergistic effect; CDI $=1$ indicates additivity; and CDI $>1$ indicates antagonism.

\section{CONFLICTS OF INTEREST}

The authors declare no conflicts of interest.

\section{REFERENCES}

1. Hess KR, Broglio KR, Bondy ML. Adult glioma incidence trends in the United States, 1977-2000. Cancer. 2004; 101:2293-9.

2. Stupp R, Mason WP, van den Bent MJ, Weller M, Fisher B, Taphoorn MJ, Belanger K, Brandes AA, Marosi C, Bogdahn U, Curschmann J, Janzer RC, Ludwin SK, et al. Radiotherapy plus concomitant and adjuvant temozolomide for glioblastoma. N Engl J Med. 2005; 352:987-96.

3. Verhaak RG, Hoadley KA, Purdom E, Wang V, Qi Y, Wilkerson MD, Miller CR, Ding L, Golub T, Mesirov JP, Alexe G, Lawrence M, O'Kelly M, et al. Integrated genomic analysis identifies clinically relevant subtypes of glioblastoma characterized by abnormalities in PDGFRA, IDH1, EGFR, and NF1. Cancer Cell. 2010; 17:98-110.

4. Phillips HS, Kharbanda S, Chen R, Forrest WF, Soriano RH, Wu TD, Misra A, Nigro JM, Colman H, Soroceanu L, Williams PM, Modrusan Z, Feuerstein BG, et al. Molecular subclasses of high-grade glioma predict prognosis, delineate a pattern of disease progression, and resemble stages in neurogenesis. Cancer Cell. 2006; 9:157-73.

5. Cancer Genome Atlas Research Network. Comprehensive genomic characterization defines human glioblastoma genes and core pathways. Nature. 2008; 455:1061-8.

6. Bhat KP, Balasubramaniyan V, Vaillant B, Ezhilarasan R, Hummelink K, Hollingsworth F, Wani K, Heathcock L, James JD, Goodman LD, Conroy S, Long L, Lelic N, et al. Mesenchymal differentiation mediated by NF-kappaB promotes radiation resistance in glioblastoma. Cancer Cell. $2013 ; 24: 331-46$.

7. Joseph JV, Balasubramaniyan V, Walenkamp A, Kruyt FA. TGF-beta as a therapeutic target in high grade gliomas- promises and challenges. Biochem Pharmacol. 2013; $85: 478-85$.

8. Zhang M, Kleber S, Röhrich M, Timke C, Han N, Tuettenberg J, Martin-Villalba A, Debus J, Peschke P, Wirkner U, Lahn M, Huber PE. Blockade of TGF-beta signaling by the TGFbetaR-I kinase inhibitor LY2109761 enhances radiation response and prolongs survival in glioblastoma. Cancer Res. 2011; 71:7155-67.

9. Lai EC. Micro RNAs are complementary to 3' UTR sequence motifs that mediate negative post-transcriptional regulation. Nat Genet. 2002; 30:363-4.

10. Sylvestre Y, De Guire V, Querido E, Mukhopadhyay UK, Bourdeau V, Major F, Ferbeyre G, Chartrand P. An E2F/ miR-20a autoregulatory feedback loop. J Biol Chem. 2007; 282:2135-43.

11. O'Donnell KA, Wentzel EA, Zeller KI, Dang CV, Mendell JT. c-Myc-regulated microRNAs modulate E2F1 expression. Nature. 2005; 435:839-43.

12. Kumar P, Luo Y, Tudela C, Alexander JM, Mendelson CR. The c-Myc-regulated microRNA-17 92 (miR-17 92) and miR-106a 363 clusters target hCYP19A1 and hGCM1 to inhibit human trophoblast differentiation. Mol Cell Biol. 2013; 33:1782-96.

13. Parsons DW, Jones S, Zhang X, Lin JC, Leary RJ, Angenendt P, Mankoo P, Carter H, Siu IM, Gallia GL, Olivi A, McLendon R, Rasheed BA, et al. An integrated genomic analysis of human glioblastoma multiforme. Science. 2008; 321:1807-12.

14. Fry DW, Harvey PJ, Keller PR, Elliott WL, Meade M, Trachet E, Albassam M, Zheng X, Leopold WR, Pryer NK, Toogood PL. Specific inhibition of cyclin-dependent kinase 4/6 by PD 0332991 and associated antitumor activity in human tumor xenografts. Mol Cancer Ther. 2004; 3:1427-1438.

15. Baughn LB, Di Liberto M, Wu K, Toogood PL, Louie T, Gottschalk R, Niesvizky R, Cho H, Ely S, Moore MA, Chen-Kiang S. A novel orally active small molecule potently induces G1 arrest in primary myeloma cells and prevents tumor growth by specific inhibition of cyclindependent kinase 4/6. Cancer Res. 2006; 66:7661-67.

16. Rahman M, Reyner K, Deleyrolle L, Millette S, Azari H, Day BW, Stringer BW, Boyd AW, Johns TG, Blot V, Duggal R, Reynolds BA. Neurosphere and adherent culture conditions are equivalent for malignant glioma stem cell lines. Anat Cell Biol. 2015; 48:25-35.

17. Pollard SM, Yoshikawa K, Clarke ID, Danovi D, Stricker S, Russell R, Bayani J, Head R, Lee M, Bernstein M, Squire JA, Smith A, Dirks P. Glioma stem cell lines expanded in adherent culture have tumor-specific phenotypes and are suitable for chemical and genetic screens. Cell Stem Cell. 2009; 4:568-80.

18. Chen L, Ye HL, Zhang G, Yao WM, Chen XZ, Zhang FC, Liang G. Autophagy Inhibition Contributes to the Synergistic Interaction between EGCG and Doxorubicin to Kill the Hepatoma Hep3B Cells. PLoS One. 2014; 9:e85771. 
19. Lu J, Getz G, Miska EA, Alvarez-Saavedra E, Lamb J, Peck D, Sweet-Cordero A, Ebert BL, Mak RH, Ferrando AA, Downing JR, Jacks T, Horvitz HR, et al. MicroRNA expression profiles classify human cancers. Nature. 2005; 435:834-8.

20. Concepcion CP, Bonetti C, Ventura A. The microRNA-17-92 family of microRNA clusters in development and disease. Cancer J. 2012; 18:262-7.

21. Woods K, Thomson JM, Hammond SM. Direct regulation of an oncogenic micro-RNA cluster by E2F transcription factors. J Biol Chem. 2007; 282:2130-2134.

22. Mao P, Joshi K, Li J, Kim SH, Li P, Santana-Santos L, Luthra S, Chandran UR, Benos PV, Smith L, Wang M, Hu B, Cheng SY, et al. Mesenchymal glioma stem cells are maintained by activated glycolytic metabolism involving aldehyde dehydrogenase 1A3. Proc Natl Acad Sci USA. 2013; 110:8644-9.

23. Lottaz C, Beier D, Meyer K, Kumar P, Hermann A, Schwarz J, Junker M, Oefner PJ, Bogdahn U, Wischhusen J, Spang R, Storch A, Beier CP. Transcriptional profiles of CD133+ and CD133- glioblastoma-derived cancer stem cell lines suggest different cells of origin. Cancer Res. 2010; 70:2030-40.

24. Günther HS, Schmidt NO, Phillips HS, Kemming D, Kharbanda S, Soriano R, Modrusan Z, Meissner H, Westphal M, Lamszus K. Glioblastoma-derived stem cell-enriched cultures form distinct subgroups according to molecular and phenotypic criteria. Oncogene. 2008;27:2897-2909.

25. Castellano DE, Rubio C, Segovia C, Porto MD, MartinezFernandez M, Otero I, Manneh R, Sepúlveda JM, Villacampa F, Paramio J. RB-independent activity of Cdk4/6 in bladder cancer. J Clin Oncol. 2016; 34; abstr e16011).

26. Comstock CE, Augello MA, Goodwin JF, de Leeuw R, Schiewer MJ, Ostrander WF Jr, Burkhart RA, McClendon AK, McCue PA, Trabulsi EJ, Lallas CD, Gomella LG, Centenera MM, et al. Targeting cell cycle and hormone receptor pathways in cancer. Oncogene. 2013; 32:5481-91.

27. Rivadeneira DB, Mayhew CN, Thangavel C, Sotillo E, Reed CA, Graña X, Knudsen ES. Proliferative suppression by CDK4/6 inhibition: complex function of the retinoblastoma pathway in liver tissue and hepatoma cells. Gastroenterology. 2010; 138:1920-11930.

28. Connell-Crowley L, Harper JW, Goodrich DW. Cyclin D1/ $\mathrm{Cdk} 4$ regulates retinoblastoma protein-mediated cell cycle arrest by site-specific phosphorylation. Mol Biol Cell. 1997; 8:287-301.

29. Liu F, Korc M. Cdk4/6 inhibition induces epithelialmesenchymal transition and enhances invasiveness in pancreatic cancer cells. Mol Cancer Ther. 2012; 11:2138-48.

30. Dean JL, Thangavel C, McClendon AK, Reed CA, Knudsen ES. Therapeutic CDK4/6 inhibition in breast cancer: key mechanisms of response and failure. Oncogene. 2010; 29:4018-32.
31. Leontieva OV, Blagosklonny MV. CDK4/6-inhibiting drug substitutes for $\mathrm{p} 21$ and $\mathrm{p} 16$ in senescence: duration of cell cycle arrest and MTOR activity determine geroconversion. Cell Cycle. 2013; 12:3063-9.

32. Leontieva OV, Demidenko ZN, Blagosklonny MV. MEK drives cyclin D1 hyperelevation during geroconversion. Cell Death Differ. 2013; 20:1241-9.

33. Qin G, Xu F, Qin T, Zheng Q, Shi D, Xia W, Tian Y, Tang Y, Wang J, Xiao X, Deng W, Wang S. Palbociclib inhibits epithelial-mesenchymal transition and metastasis in breast cancer via c-Jun/COX-2 signaling pathway. Oncotarget. 2015; 6:41794-808. http://doi.org/10.18632/ oncotarget.5993.

34. Xu T, Le TD, Liu L, Wang R, Sun B, Li J. Identifying Cancer Subtypes from miRNA-TF-mRNA Regulatory Networks and Expression Data. PLoS One. 2016; 11:e0152792.

35. Li R, Gao K, Luo H, Wang X, Shi Y, Dong Q, Luan W, You Y. Identification of intrinsic subtype-specific prognostic microRNAs in primary glioblastoma. J Exp Clin Cancer Res. 2014; 33:9.

36. Marziali G, Buccarelli M, Giuliani A, Ilari R, Grande S, Palma A, D'Alessandris QG, Martini M, Biffoni M, Pallini R, Ricci-Vitiani L. A three-microRNA signature identifies two subtypes of glioblastoma patients with different clinical outcomes. Mol Oncol. 2017; https://doi. org/10.1002/1878-0261.

37. Londin E, Loher P, Telonis AG, Quann K, Clark P, Jing Y, Hatzimichael E, Kirino Y, Honda S, Lally M, Ramratnam B, Comstock CE, Knudsen KE, et al. Analysis of 13 cell types reveals evidence for the expression of numerous novel primate- and tissue-specific microRNAs. Proc Natl Acad Sci USA. 2015; 112:E1106-15.

38. Dews M, Fox JL, Hultine S, Sundaram P, Wang W, Liu YY, Furth E, Enders GH, El-Deiry W, Schelter JM, Cleary MA, Thomas-Tikhonenko A. The myc-miR-17 92 axis blunts TGF $\{$ beta $\}$ signaling and production of multiple TGF $\{$ beta $\}$-dependent antiangiogenic factors. Cancer Res. 2010; 70:8233-46.

39. Rahme GJ, Zhang Z, Young AL, Cheng C, Bivona EJ, Fiering SN, Hitoshi Y, Israel MA. PDGF Engages an E2FUSP1 Signaling Pathway to Support ID2-Mediated Survival of Proneural Glioma Cells. Cancer Res. 2016; 76:2964-76.

40. Michaud K, Solomon DA, Oermann E, Kim JS, Zhong WZ, Prados MD, Ozawa T, James CD, Waldman T. Pharmacologic inhibition of cyclin-dependent kinases 4 and 6 arrests the growth of glioblastoma multiforme intracranial xenografts. Cancer Res. 2010; 70:3228-3238.

41. Dean JL, McClendon AK, Hickey TE, Butler LM, Tilley WD, Witkiewicz AK, Knudsen ES. Therapeutic response to CDK4/6 inhibition in breast cancer defined by ex vivo analyses of human tumors. Cell Cycle. 2012; 11:2756-61.

42. Roberts PJ, Bisi JE, Strum JC, Combest AJ, Darr DB, Usary JE, Zamboni WC, Wong KK, Perou CM, 
Sharpless NE. Multiple roles of cyclin-dependent kinase 4/6 inhibitors in cancer therapy. J Natl Cancer Inst. 2012; 104:476-87.

43. Witkiewicz AK, Cox D, Knudsen ES. CDK4/6 inhibition provides a potent adjunct to Her2-targeted therapies in preclinical breast cancer models. Genes Cancer. 2014; 5:261-72.

44. Wiedemeyer WR, Dunn IF, Quayle SN, Zhang J, Chheda MG, Dunn GP, Zhuang L, Rosenbluh J, Chen S, Xiao Y, Shapiro GI, Hahn WC, Chin L. Pattern of retinoblastoma pathway inactivation dictates response to CDK4/6 inhibition in GBM. Proc Natl Acad Sci USA. 2010; 107:11501-6.

45. Cen L, Carlson BL, Schroeder MA, Ostrem JL, Kitange GJ, Mladek AC, Fink SR, Decker PA, Wu W, Kim JS, Waldman T, Jenkins RB, Sarkaria JN. p16-Cdk4-Rb axis controls sensitivity to a cyclin-dependent kinase inhibitor PD0332991 in glioblastoma xenograft cells. Neuro Oncol. 2012; 14:870-81.
46. Finn RS, Dering J, Conklin D, Kalous O, Cohen DJ, Desai AJ, Ginther C, Atefi M, Chen I, Fowst C, Los G, Slamon DJ. PD 0332991, a selective cyclin D kinase 4/6 inhibitor, preferentially inhibits proliferation of luminal estrogen receptor-positive human breast cancer cell lines in vitro. Breast Cancer Res. 2009; 11:R77.

47. Jiao J, Huang L, Ye F, Shi M, Cheng X, Wang X, Hu D, Xie X, Lu W. Cyclin D1 affects epithelial-mesenchynal transition in epithelial ovarian cancer stem cell-like cells. Onco Targets Ther. 2013; 6:667-77.

48. Lu F, Chen Y, Zhao C, Wang H, He D, Xu L, Wang J, He X, Deng Y, Lu EE, Liu X, Verma R, Bu H, et al. Olig2Dependent Reciprocal Shift in PDGF and EGF Receptor Signaling Regulates Tumor Phenotype and Mitotic Growth in Malignant Glioma. Cancer Cell. 2016; 29:669-83. 